\title{
sciendo
}

\section{GENOME-WIDE ASSOCIATION STUDY OF WEANING TRAITS IN LORI-BAKHTIARI SHEEP}

\author{
Mohammad Almasi ${ }^{1}$, Pouya Zamani ${ }^{1}$, Seyed Ziaeddin Mirhoseini ${ }^{2}$, Mohammad Hossein Moradi ${ }^{3}$ \\ ${ }^{1}$ Department of Animal Science, Faculty of Agriculture, Bu-Ali Sina University, Hamedan, Iran \\ ${ }^{2}$ Department of Animal Science, Faculty of Agriculture, University of Guilan, Rasht, Iran \\ ${ }^{3}$ Department of Animal Science, Faculty of Agriculture, Arak University, Arak, Iran \\ •Corresponding author email: pzamani@basu.ac.ir
}

\begin{abstract}
Weaning traits, including preweaning daily gain (PWDG) and weaning weight (WW) are important economic traits, especially for meat type mammals, with high impacts on growth performance and survival rate in higher ages. This study was conducted to perform a genome-wide association study (GWAS) on weaning traits in a meat type breed of sheep. Body weight records of 7557 Lori-Bakhtiari sheep with PWDG and WW records were used to estimate breeding values (EBVs) using an animal mixed model. A total of 132 animals were selected by two-tailed selection strategy, based on EBVs for body weight and then were genotyped using Illumina 50k Ovine SNP chip. After quality control, a total of 130 animals and 41323 SNPs remained for further analyses. De-regressed estimates of breeding values were used as a pseudo-phenotype in GWAS analysis. Based on Bonferroni-adjusted P-values, five SNPs, located on chromosomes 2, 3, 4, 12 and 22 were significantly $(P<0.05)$ associated with weaning traits and accounted for $5.06 \%$ and $0.37 \%$ of total genetic variations of PWDG and WW, respectively. Two SNPs on chromosomes 2 and 3 were located near to previously reported QTLs for weaning traits. Three genes, including $A N G P T L 7$, $m T O R$ and $W D R 11$, were found within $50 \mathrm{kbp}$ distances from the significant SNPs and thus could be considered as candidate genes for weaning traits. The detected QTLs and candidate genes could be studied for construction of breeding programs for genetic improvement of growth performance in meat type sheep.
\end{abstract}

Key words: body weight, growth, quantitative trait loci (QTL), single nucleotide polymorphism (SNP), candidate gene

Genetic markers, associated with economic traits, could be used in marker assisted selection to improve profit in farm animals. Recent progresses in genomic technologies and development of ovine SNP chips, have provided the opportunity to detect genomic regions and quantitative trait loci (QTLs) associated with economic traits and possibility of genomic selection in sheep (McRae et al., 2014). 
Quantitative traits are controlled by many minor genes, imposing additive genetic effects on phenotype. However, some genes, called major genes, have higher contribution in genetic variation of the traits. Genetic markers are also used in selection for the traits for which the measurements are actually infeasible. For example, measurement of feed intake is infeasible in many farms, while economic profitability has high genetic and phenotypic correlations with different feed efficiency criteria (Zamani, 2017) and inclusion of feed efficiency in breeding objectives would generate additional genetic gain and profitability of farm animals (Zamani et al., 2008). Thus, many breeders prefer to improve profitability either by marker assisted selection or indirect selection on production traits, such as body weight in meat type animals. GWAS is a tool for detection of major genes for marker assisted selection.

Several studies using GWAS have been conducted to detect QTLs affecting body weight traits in different livestock species. For example, a QTL affecting carcass weight has been detected on bovine chromosome 6 (Setoguchi et al., 2009). In a study on Japanese Black cattle, three QTLs associated with carcass weight, were detected on chromosomes 6, 8 and 14 (Nishimura et al., 2012). Generally, few studies have been conducted on QTL mapping for production traits, especially growth and meat production traits in sheep (Al-Mamun et al., 2015). Jonas et al. (2010) reported a QTL for body weight on ovine chromosome 21. In another study, 13 SNPs, significantly associated with birth to yearling weights traits, were detected in Baluchi sheep, whereby, two SNPs, significantly associated with weaning weight, were located around two genes, DAAM1 and APIP on ovine chromosomes 7 and 15, respectively (Gholizadeh et al., 2015). Five SNPs, associated with 6-month body weight on the chromosomes 5, 6, 8, 8 and 16 and five SNPs, associated with yearling weight on chromosomes 7, 8, 13, 13 and 25 were also identified in that study (Gholizadeh et al., 2015). In a study, three SNPs on OAR1 were significantly associated with birth weight and three genes, including $R A B 6 B, T f$ and $G I G Y F 2$ were proposed as candidate genes for birth weight in Lori-Bakhtiari sheep (Ghasemi et al., 2019). In another study, a SNP located in a QTL associated with average daily gain from birth to 43 weeks of age was detected on chromosome 8 in Merino sheep (Raadsma et al., 2009). Zhang et al. (2013) reported two SNPs on OAR3 and OAR19, associated with weaning weight and preweaning gain and three SNPs associated with 6-month weight, one SNP on OAR8 and two SNPs on OAR26. Al-Mamun et al. (2015) detected 39 SNPs, significantly associated with 287-day body weight in Australian Merino sheep. Another study, conducted on Scottish Blackface lambs, revealed some SNPs on OAR6 and OAR8, significantly associated with body weights at 16 and 20 weeks of age, respectively (Riggio et al., 2013).

Sheep meat is one of the most important sources of animal protein in many countries. Weaning traits, including weaning weight and preweaning gain are important economic traits, which affect body weight gain in higher ages. Moreover, heavier lambs at weaning will have higher survival rates at higher ages (Hatcher et al., 2010). Thus, genomic scan for detection of major genes or genetic variants associated with these traits are necessary for genetic improvement of lamb production. The aim of this study was to conduct a genome-wide association study on weaning traits to 
detect possible genetic variants or major genes affecting weaning performance in a meat type breed of sheep.

\section{Material and methods}

\section{Population and data}

This study was conducted on experimental flock of Lori-Bakhtiari sheep at Shooli Breeding Station in Shahre-Kord, Iran. Lori-Bakhtiari sheep is one of the heaviest meat type breeds of sheep in the Middle East, mainly raised in western and southwestern areas of Iran. In the studied population, breeding season started from late August to late October and ewes were randomly assigned to rams. Two weeks after lambing, the suckling lambs had ad libitum access to alfalfa hay and a type of concentrate, consisting of 50\% barley, $20 \%$ wheat bran, $18 \%$ dried sugar beet pulp, $10 \%$ cotton seed meal, $1 \%$ bone meal, $0.5 \%$ salt and $0.5 \%$ vitamin-mineral supplement. Suckling lambs were weaned at $90 \pm 5$ days of age. More detailed information about the studied population has been presented by Talebi (2012).

Body weight records, lambing data and pedigree information were collected during 1989 to 2017. The pedigree included 10186 animals with 349 sires and 2364 dams, 674 animals with one unknown parent and 211 animals with both unknown parents.

The studied traits were weaning weight (WW) and preweaning daily gain (PWDG). Weaning weight was adjusted for 90 days of age as follows:

$$
W W=\left[\frac{B W_{w}-B W}{A} \times 90\right]+B W
$$

Where, $W W$ was weaning weight adjusted for 90 days of age; $B W_{w}, B W$ and $A$ were body weight at weaning, birth weight and weaning age (day), respectively.

\section{Estimation of breeding values}

Four animal mixed models (models 1-4) were compared to estimate variance components and breeding values for the studied traits. The evaluated models were as follows:

$$
\begin{aligned}
& y=X b+Z_{a} a+e \\
& y=X b+Z_{a} a+Z_{c} c+e \\
& y=X b+Z_{a} a+Z_{m} m+e \\
& y=X b+Z_{a} a+Z_{c} c+Z_{m} m+e
\end{aligned}
$$

Where, $y$ is vector of observations, $b$ is vector of fixed effects, including birth year-season (51 levels), sex (male or female), birth type (1-3) and dam age (2-7 years of age), $a, m, c$ and $e$ are vectors of random direct additive genetic, maternal 
additive genetic, maternal environmental and residual effects, respectively $X, Z_{a}, Z_{c}$ and $Z_{m}$ are incidence matrices. In the models 3 and 4 , direct additive and maternal genetic effects were considered independent of each other: $\operatorname{Cov}(a, m)=0$.

Variance components and breeding values were estimated by Average Information algorithm of Restricted Maximum Likelihood (AI-REML), using WOMBAT software (Meyer, 2007).

The models were compared by Likelihood Ratio Test (LRT) and Akaike's Information Criterion (AIC). The LRT criterion was calculated as Chisq = $2\left[\log (L)_{i}-\log (L)_{j}\right]$, where Chisq is chi-squared value, $\log (L)_{i}$ and $\log (L)_{j}$ are logarithm of likelihood in the most complex model (with the highest $\log (\mathrm{L})$ ) and the tested model, respectively. The AIC was calculated as $A I C=2 \log (L)+2 p$, where $\log (L)$ is logarithm of likelihood and $p$ is number of estimated parameters in the model. Based on the LRT, the highest $\log (\mathrm{L})$ model with significant differences with other models is considered as the best model. For non-significant LRT, a lower AIC shows a better model. Based on LRT and AIC, the model 4 had the best fit and thus was used to estimate breeding values for both WW and PWDG traits.

\section{Sampling and genotyping}

A total of 132 animals were selected for collection of blood samples. Half of the sampled animals (66 females) were selected by two-tailed selection strategy, based on EBVs for body weights (33 samples with high EBVs and 33 samples with low EBVs) and the remaining animals, including 8 males and 58 females were selected randomly. The selected animals were offspring of 120 dams and 68 sires. Blood samples (10 ml per animal) were taken from jugular vein, using EDTA containing vacuum tubes, and were stored at $-20^{\circ} \mathrm{C}$. Genomic DNA was extracted from whole blood samples, using DNP ${ }^{\mathrm{TM}} \mathrm{Kit}$ (CinnaGen Inc, Iran). All samples were genotyped using Illumina OvineSNP50 Genotyping BeadChip, which features 54,241 SNPs with an average gap size of $50.9 \mathrm{kbp}$ and a median gap size of $42.5 \mathrm{kbp}$ (Illumina Inc., CA, USA).

\section{Quality control and GWAS}

In quality control, samples with Call Rates $<99 \%$ and genotypes with GC scores $<0.6$, genotype call rates $<95 \%$, minor allele frequencies (MAF) $<0.05$ and significant Hardy-Weinberg disequilibrium $\left(\mathrm{P}<10^{-6}\right)$ were removed from the data. Moreover, the SNPs located on chromosome X were also withdrawn from the analysis. Quality control was performed using Plink 1.90 beta (Chang et al., 2015) and R software (R Core Team, 2013).

A total of 1232 SNPs located on sex chromosomes, 3081 SNPs with GC-scores $<0.6,3978$ SNPs with MAF $<0.05,1520$ with genotype call rates $<95 \%$ and one SNP with Hardy-Weinberg disequilibrium $\left(\mathrm{P}<10^{-6}\right)$ were removed from the analysis. Two samples were also withdrawn based on sample call rates $<99 \%$. After quality control, a total of 130 individuals and 41323 SNPs were used for final analyses.

De-regressed estimates of breeding values (dEBVs) were obtained, based on the method described by VanRaden et al. (2009) and then were used as a pseudo-phenotype in GWAS analysis. First five principal components (PCs) were included as 
covariates in the model to consider population structure and avoid biases due to any population stratification. The GWAS was performed using Plink 1.90 beta software (Chang et al., 2015). Q-Q and Manhattan plots were created using "qqman" package of the R software (Turner, 2014). Genetic variations of the traits, explained by the identified significant SNPs were estimated based on adjusted phenotypes, using GCTA software (Yang et al., 2011). Adjusted phenotypes were estimated as residuals of a fixed model, fitting birth year-season, sex, birth type and dam age with the levels described for fixed factors of animal mixed models (Models 1 to 4).

\section{Gene annotation}

The closest genes to the significant SNPs (after Bonferroni adjustment of P-values) within a $50 \mathrm{~kb}$ window on both sides of the SNP location were identified using sheep SNPs genome map in SNPchiMp V.3 (Nicolazzi et al., 2015) and BioMart tool of Ensembl (www.ensembl.org). Published QTLs associated with body weight traits were searched in the Animal QTL database (www.animalgenome.org/QTLdb).

\section{Results}

\section{Estimates of breeding values and genetic parameters}

The model 4 had the highest $\log (\mathrm{L})$ for both traits with significant differences with other models, based on the LRT and AIC results. The Model 4 had also the lowest AIC among total models (Table 1). Thus, the Model 4, fitting all random factors, including direct additive genetic effect and maternal genetic and environmental effects, was considered as the best model to estimate variance components and breeding values for both PWDG and WW traits.

Table 1. Fitting criteria of the studied models to estimate breeding values for WW and PWDG

\begin{tabular}{l|c|c|c|c|c|c}
\hline \multirow{2}{*}{ Model } & \multicolumn{3}{|c|}{ WW } & \multicolumn{3}{c}{ PWDG } \\
\cline { 2 - 7 } & $\log (\mathrm{L})$ & LRT Chi-Sq & AIC & LogL & LRT Chi-Sq & AIC \\
\hline 1 & -13944.15 & $178.60^{* *}$ & 27892.29 & -31644.21 & $172.22 * *$ & 63292.41 \\
2 & -13876.42 & $43.14^{* *}$ & 27758.84 & -31581.29 & $46.39 * *$ & 63168.59 \\
3 & -13860.53 & $11.36^{* *}$ & 27727.07 & -31562.61 & $9.02^{*}$ & 63131.22 \\
$4^{\dagger}$ & -13854.85 & - & 27717.71 & -31558.10 & - & 63124.20 \\
\hline
\end{tabular}

WW: weaning weight; PWDG: preweaning daily gain; $\log (\mathrm{L})$ : Logarithm of Likelihood; LRT Chi-Sq: Chisquare statistics for Likelihood Ratio Test; AIC: Akaike's Information Criterion; * and **: significant at 0.05 and 0.01 levels, respectively. $\uparrow$ : The best model based on LRT and AIC.

Genetic parameters estimated by different models are presented in Table 2. Based on the model 4, estimates of genetic parameters, including direct heritability $\left(h_{a}^{2}\right)$, maternal heritability $\left(h_{m}^{2}\right)$ and coefficient of maternal environmental effects $\left(c^{2}\right)$, were $0.12,0.12$ and 0.05 for PWDG and $0.14,0.12$ and 0.06 for WW, respectively. EBVs for PWDG and WW in the sampled animals were in ranges of -9.68 to $28.01 \mathrm{~g}$ /day and -1072 to $3205 \mathrm{~g}$, respectively. 
Table 2. Genetic parameters estimated for preweaning daily gain (PWDG) and weaning weight (WW)

\begin{tabular}{rl|c|c|c}
\hline Trait & \multicolumn{1}{c|}{ Model } & $h_{a}^{2} \pm S E$ & $h_{m}^{2} \pm S E$ & $c^{2} \pm S E$ \\
\hline PWDG & Model1 & $0.343 \pm 0.026$ & - & - \\
& Model2 & $0.118 \pm 0.023$ & $0.177 \pm 0.016$ & - \\
& Model3 & $0.188 \pm 0.026$ & - & $0.133 \pm 0.013$ \\
WW & Model4* & $0.119 \pm 0.023$ & $0.115 \pm 0.022$ & $0.053 \pm 0.017$ \\
& Model1 & $0.348 \pm 0.026$ & - & - \\
& Model2 & $0.128 \pm 0.024$ & $0.183 \pm 0.016$ & - \\
& Model3 & $0.199 \pm 0.026$ & - & $0.135 \pm 0.013$ \\
& Model4* & $0.136 \pm 0.024$ & $0.118 \pm 0.022$ & $0.056 \pm 0.017$ \\
\hline
\end{tabular}
effects.

* The best model; $h_{a}^{2}$ : direct heritability; $h_{m}^{2}:$ maternal heritability; $c^{2}$ : coefficient of maternal environmental

\section{GWAS}

Genomic inflation factor $(\lambda)$ estimated for PWDG and WW was 1.068 and 1.034, respectively. Q-Q plots of GWAS p-values for the studied traits are presented in Figure 1. Manhattan plots for GWAS of PWDG and WW are illustrated in Figure 2. Several SNPs had genome-wide P-values $<10 \mathrm{e}-5$ for association with the studied traits, but only two SNPs, including OAR2_137660266.1 (rs421003816) on chromosome 2 and OAR3_88765995.1 (rs400374454) on chromosome 3, and three SNPs, including s28298.1 (rs401389566) on chromosome 4, OAR12_45100581.1 (rs411120107) on chromosome 12 and s75433.1 (rs400287932) on chromosome 22, passed significance threshold of $\mathrm{P}<10 \mathrm{e}-6$ for PWDG and WW, respectively (Figure 2 ). These five SNPs were also significant, after Bonferroni adjustment of $\mathrm{P}$-values $(\mathrm{P}<0.05)$. More information about the significant SNPs is presented in Table 3. The significant SNPs accounted for $5.06 \%$ and $0.37 \%$ of total genetic variations of PWDG and WW, respectively (Table 3 ).

Table 3. Significant SNPs associated with PWDG and WW

\begin{tabular}{c|c|c|c|c|c|c|c}
\hline Trait & SNP & Chr. & Location (bp) & $\begin{array}{c}\text { Genome wide } \\
\text { P-value }\end{array}$ & Bonf P & GVE (\%) & $\alpha$ \\
\hline PWDG & rs421003816 & 2 & 129295009 & $7.970 \mathrm{e}-07$ & 0.0329 & 1.45 & 0.030 \\
& rs400374454 & 3 & 83846136 & $1.150 \mathrm{e}-07$ & 0.0048 & 3.61 & 0.025 \\
WW & rs401389566 & 4 & 76527529 & $2.720 \mathrm{e}-07$ & 0.0112 & 0.08 & 0.441 \\
& rs411120107 & 12 & 40594072 & $2.457 \mathrm{e}-07$ & 0.0102 & 0.19 & -1.020 \\
& rs400287932 & 22 & 39852684 & $4.463 \mathrm{e}-07$ & 0.0184 & 0.10 & -0.109 \\
\hline
\end{tabular}

WW: weaning weight; PWDG: preweaning daily gain; Bonf p: Bonferroni adjusted P-values; GVE: genetic variance explained; $\alpha$ : allele substitution effect for the minor frequency allele (g/day for PWDG and $g$ for WW). 

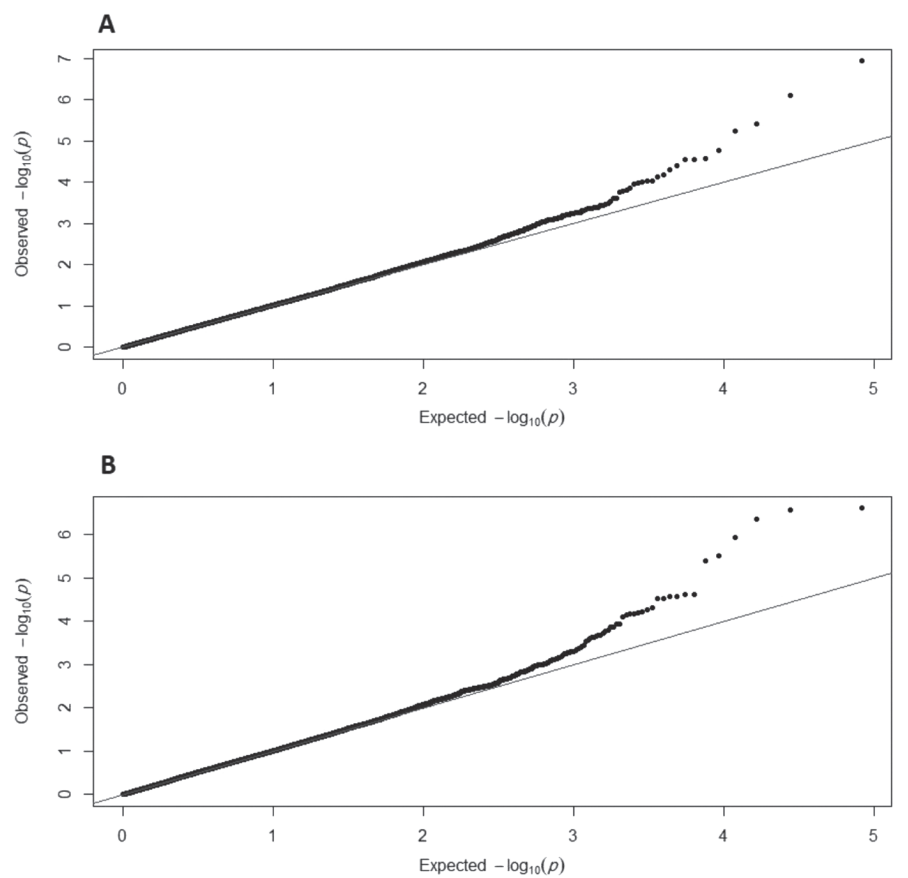

Figure 1. Q-Q plots of $-\log 10$ (P-values) for preweaning daily gain (graph A) and weaning weight (graph B). The dots represent $-\log 10$ (P-value) of the single nucleotide polymorphisms (SNPs) and the $45^{\circ}$ line represents the expected values under the null hypothesis for no association
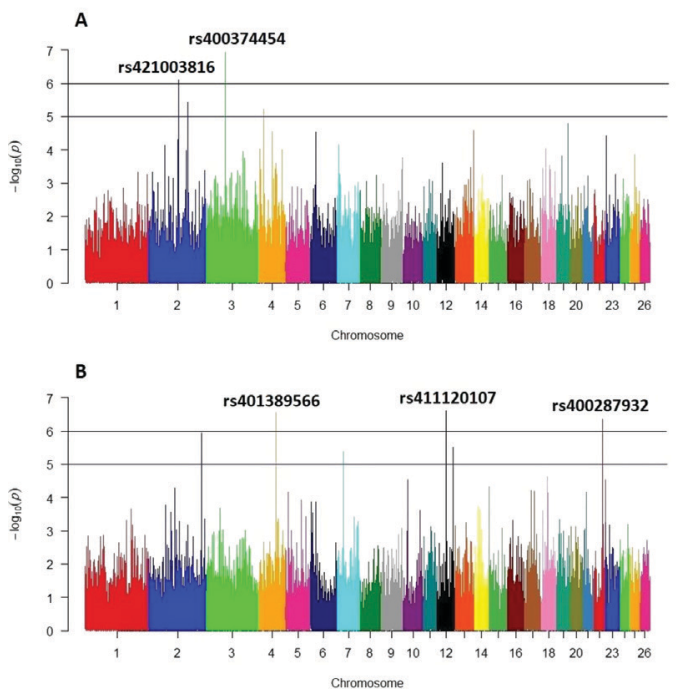

Figure 2. Manhattan plots of genome-wide P-values for preweaning daily gain (Graph A) and weaning weight (Graph B). Genomic positions with chromosome numbers are represented on the $\mathrm{X}$-axis and $-\log 10$ (P-values) are shown on the Y-axis. Lower and upper horizontal lines indicate significance thresholds of $10 \mathrm{e}-5$ for genome-wide P-value and 0.05 for Bonferroni adjusted P-values, respectively 


\section{QTLs and genes annotation}

The significant SNPs (rs421003816, rs400374454, rs401389566, rs411120107 and rs400287932) were located near to several body weight associated QTLs, reported in Animal QTL database (www.animalgenome.org/QTLdb). Based on BioMart-Ensembl database, four genes were found within $50 \mathrm{kbp}$ distances from the significant SNPs, associated with WW on chromosomes 4, 12 and 22 (Table 4). The SNP rs401389566 was located in a 3723 bp distance from $R A M P 3$, receptor activity modifying protein 3, on chromosome 4 (Figure 3). The SNP rs411120107 was located within $m T O R$, mechanistic target of rapamycin kinase and $625 \mathrm{bp}$ distance from ANGPTL7, angiopoietin like 7, on chromosome 12 (Figure 4) and the last significant SNP, rs400287932 had a 7548 bp distance from WDR11, WD Repeat Domain 11, on chromosome 22 (Figure 5). Other significant SNPs, rs421003816 and rs400374454 were not located in $50 \mathrm{kbp}$ distances from any other gene (Table 4).

Table 4. Genes annotation within $50 \mathrm{kbp}$ distances from the significant SNPs

\begin{tabular}{c|c|c|c|c|c|c|c}
\hline SNP & Chr. & SNP position (bp) & Gene & $\begin{array}{c}\text { Gene start } \\
(\mathrm{bp})\end{array}$ & $\begin{array}{c}\text { Gene end } \\
(\mathrm{bp})\end{array}$ & Strand & $\begin{array}{c}\text { Distance to } \\
\text { SNP }(\mathrm{bp}) *\end{array}$ \\
\hline rs421003816 & 2 & 129295009 & - & - & - & - & - \\
rs400374454 & 3 & 83846136 & - & - & - & - & - \\
rs401389566 & 4 & 76527529 & RAMP3 & 76531252 & 76535198 & $\mathrm{R}$ & -3723 \\
rs411120107 & 12 & 40594072 & ANGPTL7 & 40588687 & 40593447 & $\mathrm{R}$ & 625 \\
& & & mTOR & 40533385 & 40657651 & $\mathrm{~F}$ & Within \\
rs400287932 & 22 & 39852684 & WDR11 & 39787927 & 39845136 & $\mathrm{~F}$ & 7548 \\
\hline
\end{tabular}

* Genes with negative and positive distances are located in downstream and upstream of the SNPs, respectively; F and R: forward and reverse strands, respectively.

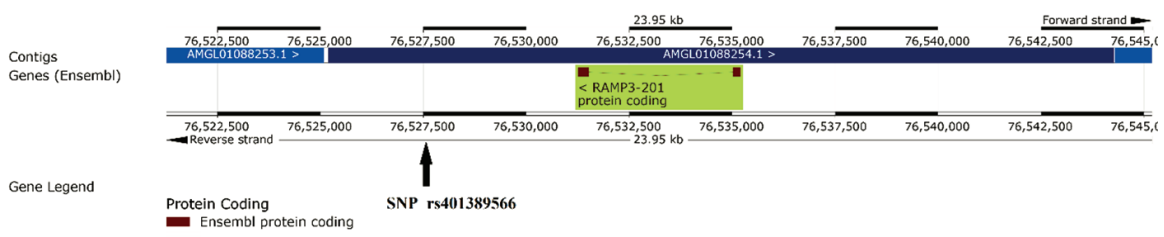

Figure 3. Location of RAMP3 near to the SNP rs401389566 on $76.528 \mathrm{Mbp}$ of OAR4; the significant SNP is marked by an arrow

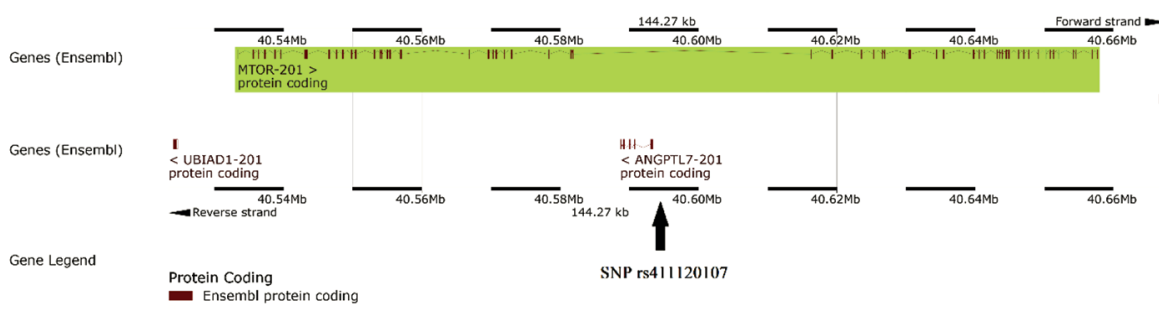

Figure 4. Locations of $m$ TOR and ANGPTL7 genes around the SNP rs411120107 on 40.594 Mbp of OAR12; the significant SNP is marked by an arrow 


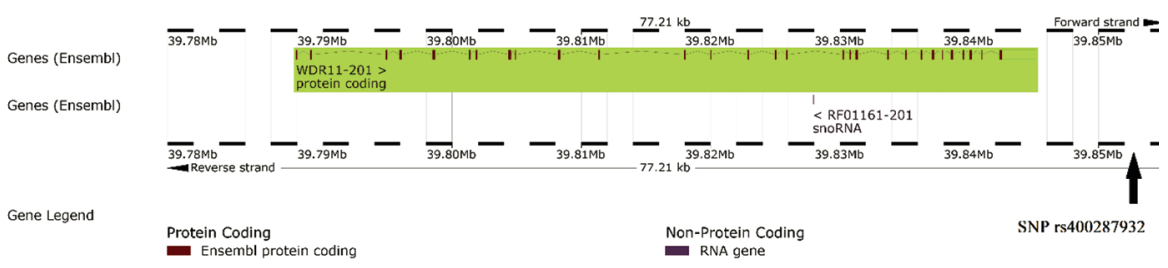

Figure 5. Location of WDR11 gene near to the SNP rs400287932 on 39.853 Mbp of OAR22; the significant SNP is marked by an arrow

\section{Discussion}

Generally, quantitative traits, such as body weight and body weight gain traits are influenced by considerably large number of loci. Thus, it would be expected that several QTLs could be found for different quantitative traits. Several QTLs and candidate genes have been reported for quantitative traits in sheep, either reproduction (Abdoli et al., 2016, 2018, 2019) or body weight traits (Matika et al., 2016; Wang et al., 2016; Ghasemi et al., 2019). Based on Sheep QTL category of Animal QTL database (www.animalgenome.org/QTLdb), no QTL have been reported for pre-weaning daily gain, but some QTLs associated with weaning weight have been reported on 128.7-128.8 Mbp of OAR2, 79.3 Mbp and 213.7 Mbp of OAR3, 65.4 Mbp of OAR4, 68.4 Mbp of OAR7, 13.6 Mbp of OAR9, 63.5 Mbp of OAR15, 51.3 Mbp of OAR19 and 34.3 Mbp of OAR24.

Wang et al. (2016) identified a SNP, associated with weaning weight, in promotor of myostatin gene $(M S T N)$, on $128.7 \mathrm{Mbp}$ of OAR2, which is near to the SNP rs421003816 in the present study. In a study on Sunit, German mutton and Dorper breeds, a SNP on 213.9 Mbp of OAR3 was significantly associated with PWDG and WW (Zhang et al., 2013). Based on another study (Ma et al., 2016), a QTL on 79.3 Mbp of OAR3 has been reported for 4-month body weight in Animal QTL database (www.animalgenome.org/QTLdb), which is near to the significant SNP rs400374454 on $83.8 \mathrm{Mbp}$ of OAR3, in this study. In Awassi-Merino backcross sheep, two QTLs on 10.9-11.1 Mbp of OAR3 and 71.5-71.6 Mbp of OAR12 were associated with average daily gain during birth to 43 weeks of age and two QTLs on 15.7-43.7 Mbp of OAR21 and 26.0-32.4 Mbp of OAR24 were both associated with body weights at 43,56 and 83 weeks of age and average daily gains in 0-43, 43-56 and 56-83 weeks of age. In that study, two more QTLs, located on 14.1-14.2 Mbp of OAR21 and 9.1-26.0 Mbp of OAR24 were significantly associated with average daily gain from birth to 43 weeks of age (Raadsma et al., 2009). Matika et al. (2016) detected two QTLs, located on 0.3-6.4 Mbp of OAR22 and 24.0-31.2 Mbp of OAR24, associated with live weight at 24 weeks of age in Scottish Blackface lambs. In a more recent study on Texel sheep, four SNPs on 65.3 and $65.4 \mathrm{Mbp}$ of OAR4, 13.6 Mbp of OAR9 and 34.3 Mbp of OAR24 were associated with weaning weight (Armstrong et al., 2018). The reported QTL on OAR4 by Armstrong et al. (2018) is, to some extent, near to the significant SNP on OAR4 in the present study. 
Based on Oar_v3.1 dataset in BioMart tool of Ensembl genome browser (www. ensembl.org), the observed significant SNPs on OAR2 and OAR3 were not located in $50 \mathrm{kbp}$ distances from known genes. However, the SNP rs421003816, was located near to myostatin (MSTN) gene on $129 \mathrm{Mbp}$ of OAR2 (www.ncbi.nlm.nih. gov/gene/443449). Significant effects of MSTN on body weight and skeletal muscle traits have been well known (Bellinge et al., 2005). Other significant SNPs on chromosomes 4, 12 and 22, were located in $50 \mathrm{kbp}$ distances from some genes, including RAMP3, ANGPTL7, mTOR and WDR11 (Table 4).

The RAMP3 gene encodes a member of the RAMP family of single-transmembrane-domain proteins, called receptor activity modifying proteins (RAMP1, RAMP2 and RAMP3). The RAMPs are needed to transport calcitonin-receptor-like (CRL) receptor to the plasma membrane which can function as either a calcitoningene-related peptide (CGRP) receptor or an adrenomedullin (AM) receptor, depending on expression of the RAMP family member. In presence of the RAMP3 protein, the CRLR acts as an adrenomedullin receptor. RAMPs were first identified as enhancers of cell surface expression of the calcitonin-like receptor (CLR), but now they are known to alter trafficking, signaling and pharmacology in a receptor-dependent manner (Hay and Pioszak, 2016). In one of rare evidences for association of RAMP3 with body weight trait, it has been found that $R A M P 3$ may have an important effect on mice body weight, but physiological mechanisms accounting for this phenotype have not been resolved (Dackor et al., 2007).

Angiopoietin-like proteins (ANGPTLs) belong to an eight-member family of proteins with structures similar to the angiopoietins and various functions in developmental, physiological and pathophysiological processes, including functional roles in lipid metabolism, inflammation, hematopoietic stem cell activity and cancer cell invasion (Santulli, 2014). Moreover, the ANGPTL proteins, including angiopoietin-like protein 7 ( $A N G P T L 7$ ), have shown to be involved in controlling angiogenic processes of endochondral bone formation (Johannessen et al., 2007), obesity and fasting blood triglyceride plasma (Abu-Farha et al., 2017). Thus, the ANGPTL7 may affect early growth performance and could be considered as a candidate gene for growth traits in growing animals.

The mechanistic target of rapamycin (mTOR) is a protein kinase that is a specific target of the natural compound rapamycin (Oh and Jacinto, 2011). The mTOR is evolutionarily conserved in all eukaryotes and is involved in many fundamental metabolic and physiological processes, including lipid metabolism, not only lipogenesis and lipolysis, but also adipogenesis (Lamming and Sabatini, 2013). It has been found that the mTOR controls cell growth, in part by regulating p70 S6 kinase $\alpha(\mathrm{p} 70 \alpha)$, eukaryotic initiation factor 4E binding protein 1 (4EBP1) and raptor, a mTOR binding protein which also binds 4EBP1 and p70 $\alpha$ (Hara et al., 2002). The $\mathrm{mTOR}$ is master regulator of cell growth and metabolic state in response to nutrients, growth factors and many extracellular cues (Mao and Zhang, 2018) and plays important roles in actin cytoskeleton reorganization and cell migration, autophagy, proliferation, mitochondrial respiration and protein synthesis and maturation (Oh and Jacinto, 2011). In addition to central regulation role of $m$ TOR signaling pathway on cell growth and metabolism, its deregulation has been implicated in several dis- 
eases such as cancer, diabetes, obesity, neurological diseases and genetic disorders (Li et al., 2014). It seems that the mTOR could be considered as another candidate gene for growth traits.

WD Repeat Domain 11 (WDR11) is a protein coding gene on OAR22. Several functions, including tumor suppression, transcriptional regulation, ricin trafficking and control of viral assembly have been proposed for WDR11. Moreover, WDR11 localizes to the nucleus, to autophagosomes and to the trans-Golgi network and facilitates the tethering of AP-1-derived vesicles (Navarro Negredo et al., 2018). In addition to transcriptional regulation function, WDR11 interacts with some transcription factors (Kim et al., 2010). It has been found that some mutations in a number of genes, encoding transcription factors, such as WDR11 are associated with pituitary dysfunction and abnormal development of pituitary gland (Di Iorgi et al., 2016). Thus, WDR11 may be considered as a candidate gene for body weight traits for future studies.

\section{Conclusion}

The significant SNPs accounted for $5.06 \%$ and $0.37 \%$ of total genetic variations of PWDG and WW, respectively. Among the detected SNPs, two SNPs on 129.3 Mbp of OAR2 and $83.8 \mathrm{Mbp}$ of OAR3 were located near to previously reported QTLs for weaning traits. Other SNPs, located on 76.5 Mbp of OAR4, 40.6 Mbp of OAR12 and 39.9 Mbp of OAR22 were new locations for QTLs, associated with weaning traits. These SNPs however, were located near to some genes, associated with body weight traits. Based on the known functions of the genes, located within $50 \mathrm{kbp}$ distances from the significant SNPs, ANGPTL7, $m T O R$ and WDR11 genes could be considered as candidate genes for weaning traits. However, more studies on the proposed QTLs and candidate genes may provide facilities for genetic improvement of weaning performance and other body weight traits in sheep.

\section{Conflict of interest}

The authors declare that they do not have any conflict of interest.

\section{Acknowledgements}

Fund of this study was provided by Bu-Ali Sina University and University of Guilan. The authors appreciate Dr. Ramin Abdoli, Bu-Ali Sina University; Dr. Rostam Abdollahi-Arpanahi, University of Tehran and Prof. Cedric Gondro, Michigan State University for their kind advices and cooperation.

\section{References}

Abdoli R., Zamani P., Mirhoseini S.Z., Ghavi Hos sein-Zadeh N., Nadri S. (2016). A review on prolificacy genes in sheep. Reprod. Domest. Anim., 51: 631-637.

Abdoli R., Mirhoseini S.Z., Ghavi Hossein-Zadeh N., Zamani P., Gondro C. (2018). Genome-wide association study to identify genomic regions affecting prolificacy in LoriBakhtiari sheep. Anim. Genet., 49: 488-491. 
Abdoli R., Mirhoseini S.Z., Ghavi Hossein-Zadeh N., Zamani P., Moradi M.H., Ferdosi M.H., Gondro C. (2019). Genome-wide association study of first lambing age and lambing interval in sheep. Small Rumin. Res., 178: 43-45.

A bu-Farha M., Cherian P., A $1-$ Khairi I., Madhu D., Tiss A., Warsam S., A lhubail A., Srir a m an D., A l-Refa e i F., A bu baker J. (2017). Plasma and adipose tissue level of angiopoietin-like 7 (ANGPTL7) are increased in obesity and reduced after physical exercise. PLoS One, 12: e0173024.

A 1 - M a m u n H.A., Kw an P., C 1 ark S.A., Ferdosi M.H., Tella m R., Gondro C. (2015). Genome-wide association study of body weight in Australian Merino sheep reveals an orthologous region on OAR6 to human and bovine genomic regions affecting height and weight. Genet. Sel. Evol., 47: 66.

Armstrong E., Ciappesoni G., Iriarte W., Da Silva C., Macedo F., Navajas E.A., Brito G., S an Julián R., Gimeno D., Postiglioni A. (2018). Novel genetic polymorphisms associated with carcass traits in grazing Texel sheep. Meat Sci., 145: 202-208.

B e 1 ling e R.H.S., L ib er le s D.A., I a s ch i S.P.A., O 'B ri en P.A., T a y G.K. (2005). Myostatin and its implications on animal breeding: a review. Anim. Genet., 36: 1-6.

Chang C.C., Chow C.C., Telli er L.C.A.M., Vattikuti S., Purcell S.M., Le e J.J. (2015). Second-generation PLINK: Rising to the challenge of larger and richer datasets. Gigascience, 4: $1-16$.

D a c k or R., Fritz-S ix K., S mithies O., C a r on K. (2007). Receptor activity-modifying proteins 2 and 3 have distinct physiological functions from embryogenesis to old age. J. Biol. Chem., 282: 18094-18099.

Di Iorgi N., Morana G., A 11 eg r i A.E.M., N a poli F., G astaldi R., Calcagno A., Pat t i G., L o c h e S. (2016). Classical and non-classical causes of GH deficiency in the paediatric age. Best Pract. Res. Clin. Endocrinol. Metab., 30: 705-736.

Ghas e mi M., Z a m a ni P., Vat a $\mathrm{kh}$ ah M., A bd oli R. (2019). Genome-wide association study of birth weight in sheep. Animal, 13: 1797-1803.

Gholizad eh M., Rahim i-Mianji G., N e jat i - J a vare mi A. (2015). Genomewide association study of body weight traits in Baluchi sheep. J. Genet., 94: 143-146.

Hara K., Maruki Y., Long X., Yoshino K., Oshiro N., Hidayat S., Tokunaga C., Avruch J., Yonezawa K. (2002). Raptor, a binding partner of target of rapamycin (TOR), mediates TOR action. Cell, 110: 177-189.

Hatcher S., E p plest on J., Th or n berry K.J., Wat t B. (2010). High Merino weaner survival rates are a function of weaning weight and positive post-weaning growth rates. Anim. Prod. Sci., 50: 465-472.

H a y D.L., P i o s z a k A.A. (2016). Receptor activity-modifying proteins (RAMPs): new insights and roles. Annu. Rev. Pharmacol. Toxicol., 56: 469-487.

Johannessen M.K., Skretting G., Ytrehus B., Røed K.H. (2007). Neonatal growth cartilage: equine tissue specific gene expression. Biochem. Biophys. Res. Commun., 354: 975-980.

J o n a s E., T h o m s o n P.C., R a a d s m a H.W. (2010). Genome-wide association study and fine mapping of QTL on OAR 21 for body weight in sheep. Proc. 9th World Congress on Genetics Applied to Livestock Production, Leipzig, Germany.

Kim H.G., Ahn J.W., Kurth I., U $11 \mathrm{~m}$ an n R., Kim H.T., Kulharya A., H a K.S., I to kawa Y., Meliciani I., Wenzel W., Lee D., Rosenberger G., Ozata M., Bick D.P., Sherins R.J., Nagase T., Tekin M., Kim S.H., Kim C.H., Ropers H.H., Guse11 a J.F., Ka $1 \mathrm{scch}$ e u e r V., Ch o i C.Y., L a y m a n L.C. (2010). WDR11, a WD protein that interacts with transcription factor EMX1, is mutated in idiopathic hypogonadotropic hypogonadism and Kallmann syndrome. Am. J. Hum. Genet., 87: 465-479.

Lamming D.W., Sabatini D.M. (2013). A central role for mTOR in lipid homeostasis. Cell Metab., 18: 465-469.

L i J., K i m S.G., B l e n i s J. (2014). Rapamycin: one drug, many effects. Cell Metab., 19: 373-379.

Ma X., Guan L., Xu an J., Wang H., Yuan Z., Wu M., Li u R., Zhu C., We i C., Zha o F. (2016). Effect of polymorphisms in the CAMKMT gene on growth traits in Ujumqin sheep. Anim. Genet., 47: 618-622. 
Ma o Z., Z hang W. (2018). Role of mTOR in glucose and lipid metabolism. Int. J. Mol. Sci., 19: 2043.

Matika O., Riggio V., Anselme-Moizan M., Law A.S., Pong-Wong R., Archibald A.L., B is h op S.C. (2016). Genome-wide association reveals QTL for growth, bone and in vivo carcass traits as assessed by computed tomography in Scottish Blackface lambs. Genet. Sel. Evol., 48: 11.

McRae K.M., Mcewan J.C., Dodds K.G., Gemmell N.J. (2014). Signatures of selection in sheep bred for resistance or susceptibility to gastrointestinal nematodes. BMC Genomics, 15: 637.

M e y e r K. (2007). WOMBAT - A tool for mixed model analyses in quantitative genetics by restricted maximum likelihood (REML). J. Zhejiang Univ. Sci. B, 8: 815-821.

Navarro Negredo P., Edgar J.R., Manna P.T., Antrobus R., Robinson M.S. (2018). The WDR11 complex facilitates the tethering of AP-1-derived vesicles. Nat. Commun., 9: 596.

Nicolazzi E.L., Caprera A., Nazzicari N., Cozzi P., Strozzi F., Lawley C., Pirani A., S o ans C., Brew F., Jorjani H., Evans G., S impson B., Tos ser-Klopp G., Brauning R., William s J.L., S tella A. (2015). SNPchiMp v. 3: integrating and standardizing single nucleotide polymorphism data for livestock species. BMC Genomics, 16: 283.

Nishimura S., Watanabe T., Mizoshita K., Tatsuda K., Fujita T., Watanabe N., S u g i moto Y., Tak as ug a A. (2012). Genome-wide association study identified three major QTL for carcass weight including the PLAG1-CHCHD7 QTN for stature in Japanese Black cattle. BMC Genet., 13: 40.

Oh W.J., Jacinto E. (2011). mTOR complex 2 signaling and functions. Cell Cycle, 10: $2305-2316$

R Core Team (2013). R: A language and environment for statistical computing, R Foundation for Statistical Computing. Citeseer, Vienna, Austria.

Raadsma H.W., Thomson P.C., Zenger K.R., Cavanagh C., Lam M.K., Jon as E., J on es M., A t t a rd G., P a lmer D., Ni cholas F.W. (2009). Mapping quantitative trait loci (QTL) in sheep. I. A new male framework linkage map and QTL for growth rate and body weight. Genet. Sel. Evol., 41: 34.

Riggio V., Matika O., Pong-Wong R., Stear M.J., Bishop S.C. (2013). Genome-wide association and regional heritability mapping to identify loci underlying variation in nematode resistance and body weight in Scottish Blackface lambs. Heredity (Edinb), 110: 420-429.

S antulli G. (2014). Angiopoietin-like proteins: a comprehensive look. Front. Endocrinol. (Lausanne), 5: 6 .

S et oguchi K., Furuta M., Hirano T., Nagao T., Watanabe T., Sugimoto Y., Takas u g a A. (2009). Cross-breed comparisons identified a critical 591-kb region for bovine carcass weight QTL (CW-2) on chromosome 6 and the Ile-442-Met substitution in NCAPG as a positional candidate. BMC Genet., 12: 43.

Ta 1 e bi M.A. (2012). Feed intake, feed efficiency, growth and their relationship with Kleiber ratio in Lori-Bakhtiari lambs. Arch. Zootech., 15: 33-39.

Turne r S.D. (2014). qqman: an R package for visualizing GWAS results using QQ and manhattan plots. BioRxiv, 3: 5165.

Van Rad en P.M., Van Tas se 11 C.P., Wiggan s G.R., S on st e gard T.S., S chnabel R.D., Taylor J.F., Schenkel F.S. (2009). Invited review: Reliability of genomic predictions for North American Holstein bulls. J. Dairy Sci., 92: 16-24.

Wang J., Z h o u H., H u J., Li S., L u o Y., H i ck ford J.G.H. (2016). Two single nucleotide polymorphisms in the promoter of the ovine myostatin gene (MSTN) and their effect on growth and carcass muscle traits in New Zealand Romney sheep. J. Anim. Breed. Genet., 133: 219-226.

Yan g J., L e e S.H., G o d d a r d M.E., Visscher P.M. (2011). GCTA: A tool for genome-wide complex trait analysis. Am. J. Hum. Genet., 88: 76-82.

$\mathrm{Z}$ a m a n i P. (2017). Statistical properties of proportional residual energy intake as a new measure of energetic efficiency. J. Dairy Res., 84: 248-253.

Zamani P., Miraei-A shtiani S.R., Moham madi H. (2008). Genetic parameters of residual energy intake and its correlations with other traits in Holstein dairy cattle. Turk. J. Vet. Anim. Sci., 32: $255-261$. 
Zhang L., Li u J., Zha o F., Ren H., Xu L., Lu J., Zhang S., Zhang X., We i C., Lu G., $\mathrm{Z}$ h eng Y., D u L. (2013). Genome-wide association studies for growth and meat production traits in sheep. PLoS One, 8: e66569.

Received: 8 IX 2019

Accepted: 9 I 2020 